\title{
$\mathrm{PbTiO}_{3}$ 微晶在凝胶玻璃中的生长及其尺寸效应*
}

\author{
姚 奎 周歧发 张良堂 姚 喜 \\ (西安交通大学电子材料研究室, 西安 710049)
}

\section{关键词钛酸铅溶胶凝胶工艺微晶玻璃 尺寸效应}

以铁电相为主晶相的微晶玻璃在电光效应, 高介介质, 以及调节玻璃的热胀系数等方面具 有重要的应用价值, 自 60 年代就引起人们关注 ${ }^{[1,2]}$. 随着当前纳米铁电微晶和精细复合技术 研究的深人 ${ }^{[3,4]}$, 迫切需要克服铁电超微粒子的团聚, 获得尺寸易控的纳米铁电微晶以及制备 新的复合体系. 若能利用玻璃体中的受限环境原位析出尺寸易控的 $\mathrm{PbTiO}_{3}$ 超微晶粒对该领 域的研究有重要意义.

但是, 采用传统工艺制备 $\mathrm{PbTiO}_{3}$ 铁电微晶玻璃要求 $1350^{\circ} \mathrm{C}$ 以上的高温融熔过程和极快速 的淬火处理, 工艺条件较苛刻, 制备的样品组成有限, 内应力较大, 而且存在着高温下 $\mathrm{Pb}$ 的强烈 挥发. 作者在以前工作的基础上 ${ }^{[5,6}$, 采用新颖的溶胶凝胶工艺, 成功地在不同组成的 $\mathrm{Pb}-\mathrm{Ti}-\mathrm{Si}$ 凝 胶中析出了纯净的 $\mathrm{PbTiO}_{3}$ 铁电纳米微晶, 并进一步探讨了原位析出的 $\mathrm{PbTiO}_{3}$ 微晶的尺寸效 应.

\section{1 实验过程}

将三水醋酸铅溶于乙二醇乙醚中, 加热脱去结晶水, 在强烈摚拌下缓慢向其中加人钛酸 四丁酯 $(\mathrm{Pb} / \mathrm{Ti}=1 / 1)$, 并升高加热温度. 随着反应的进行, 体系逐渐转变成具有 $\mathrm{Pb}-\mathrm{Ti}$ 复令结 构的金黄色溶液. 待其降至室温后, 与 TEOS 混合, 获得 Pb-Ti-Si 溶胶. 溶胶中的元素逐渐 吸收空气中水分发生水解聚合反应, 几十小时后, 形成清澈的均匀凝胶. 凝胶在室温下缓慢地 自然干燥, 成为具有一定强度的透明干凝胶. 将干凝胶置人马弗 (muffle) 炉内缓慢加热, 随处 理温度的升高, 分别获得 $\mathrm{Pb}-\mathrm{Ti}$-Si 凝胶玻璃和微晶玻璃.

$\mathrm{Pb}-\mathrm{Ti}$-Si 玻璃网络结构的红外研究采用 Nicolet 公司的 SDX-FTIR 仪. $\mathrm{PbTiO}_{3}$ 微晶晶格 结构和晶粒尺寸的计算采用日本理学的 $D / m a x-2400 X$ 光衍射仪. 热分析采用杜邦公司的 DTA1600 和 910DSC. 形貌观察利用日立公司分辨率较高的 S-2700 SEM.

\section{2 结果与讨论}

利用 IR 谱分析了含 $\mathrm{PbO}-\mathrm{TiO}_{2}$ (下简称 PT) $42 \%$ 的 $\mathrm{PbO}-\mathrm{TiO}_{2}-\mathrm{SiO}_{2}$ (下简称 PTS) 溶胶和 凝胶的结构随温度的变化. 结果表明, PTS 溶体中原始反应物的有机基团的振动峰较强, 而 在干凝胶中绝大部分有机基团的吸收峰已显著减弱 (醋酸根吸收峰要到 $350^{\circ} \mathrm{C}$ 左右消失), 并 形成了以 $\mathrm{Si}-\mathrm{O}-\mathrm{Si}$ 键为主体的网络结构, 同时也产生了 $\mathrm{Si}-\mathrm{O}-\mathrm{Ti}$ 键. 随着温度升高, $\mathrm{Si}-\mathrm{O}-\mathrm{Si}$ 键 加强, $\mathrm{Si}-\mathrm{O}-\mathrm{Ti}$ 键减弱, 在 $600^{\circ} \mathrm{C}$ 以上 $\mathrm{Si}-\mathrm{O}-\mathrm{Ti}$ 键逐渐消失. 表明较高温度下 $\mathrm{Ti}$ 组元逐渐从玻

\footnotetext{
1994-05-07 收稿, 1994-09-12 收修改稿

*国家“八六三”高技术新材料基金资助项目
} 
璃网络中析出. IR 谱上较强的 $\mathrm{Pb}-\mathrm{O}$ 键的吸收峰在 $700^{\circ} \mathrm{C}$ 以上出现.

图 1 是含 PT $42 \%$ 的 PTS 经不同温度处理后的 XRD 谱. 在如此低的 PT 浓度下析出纯 净的钻钢矿结构在前人的工作中未见报道. 图 2 是不同 PT 含量的 PTS 和 XRD 谱. 尽管纯 PT 凝胶粉末能在 $488^{\circ} \mathrm{C}$ 直接形成钙钛矿结构 ${ }^{[9}$, 但 PTS 在析出钙钛矿结构以前, 首先形成介

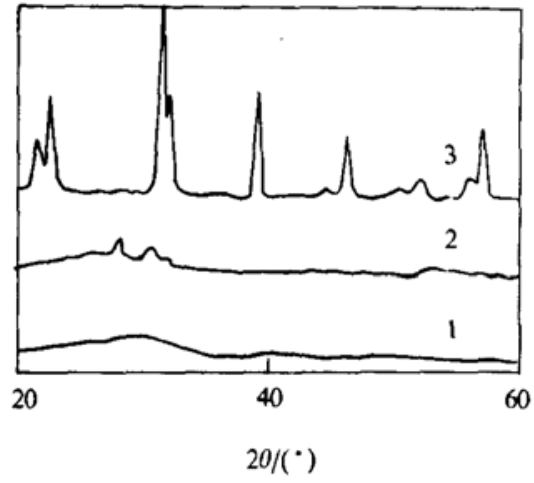

图 1 不同温度处理的 PTS 的 XRD 谱 1 为 $600^{\circ} \mathrm{C}, 2 \mathrm{~h} ; 2$ 为 $700^{\circ} \mathrm{C}, 2 \mathrm{~h} ; 3$ 为 $800^{\circ} \mathrm{C}, 2 \mathrm{~h}$

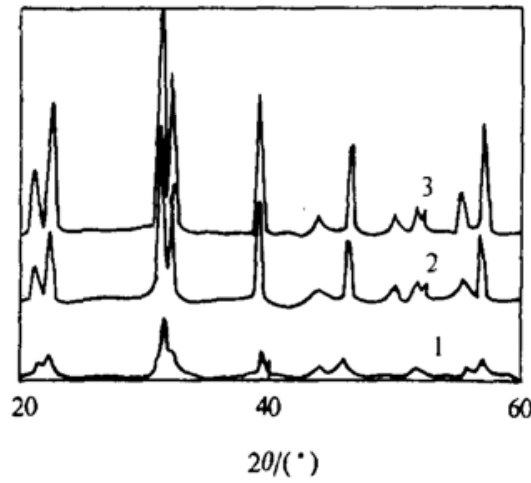

图 2 不同 PT 含量的 PTS 的 XRD 谱 $\left(800^{\circ} \mathrm{C}, 2 \mathrm{~h}\right)$

1 为 $42 \% \mathrm{PT} ; 2$ 为 $73 \% \mathrm{PT} ; 3$ 为 $85 \% \mathrm{PT}$

稳过渡相. 当 PT 含量高时, 此过渡相为完整的 $\mathrm{Pb}_{2} \mathrm{Ti}_{2} \mathrm{O}_{6}$ 焦绿石相; 当 PT 含量低时, 过渡相为 某个远未充分发展的未知相. 这些过渡相均能在更高温度下完全转化为钻钠矿结构. 结合 DTA 的结果发现, PT 含量越低, 焦绿石相的形成温度和向钻钛矿结构的转变温度越高. 当 PT 含量为 $85 \%$ 和 $42 \%$ 时, PTS 形成过渡相结构的温度分别为 $550{ }^{\circ} \mathrm{C}$ 和 $610{ }^{\circ} \mathrm{C}$, 向钙钢矿结构的 转变温度为 $648^{\circ} \mathrm{C}$ 和 $710^{\circ} \mathrm{C}$ 以上. 含 PT $30 \%$ 的 PTS 在 $850^{\circ} \mathrm{C}$ 下处理仍未能获得钻钛矿结构.

PT 晶粒结构、尺寸的精确测定及其小尺寸效应的主要研究结果概括于表 1 中. X 衍射仪 经 $\mathrm{Si}$ 标样校正. 晶粒尺寸数据的计算是根据 Scherrer 原理, 采用 Gauss 近似获得. 并利用 计算机进行峰分离处理, 扣除仪器宽度. 表 1 中选择 (200) 晶面上晶粒尺寸计算结果作为代 表, (002) 晶面上晶粒尺寸数据较 (200) 晶面小 $30 \% \sim 40 \%$, 且数据分散性较大. 作者认为, 这 是因为纳米微晶的尺寸效应和所受应力作用造成 PT 晶粒 $c$ 轴长度有较大变化所致. 表 1 数 据表明, $800^{\circ} \mathrm{C}$ 下处理 $2 \mathrm{~h}$ 的含 $42 \% \mathrm{PT}$ 的 PTS 中析出了尺寸近 $20 \mathrm{~nm}$ 的 $\mathrm{PbTiO}_{3}$ 微晶, 且随着 PTS 中 PT 含量的增加, 相同热处理条件下析出的 PT 微晶的尺寸增大. 可见, 利用凝胶玻璃 体内的受限环境, 在保证钙钛矿结构完整的同时, 比 $\mathrm{PbTiO}_{3}$ 微粉制备技术获得了尺寸更易控 制的超微粒子. SEM 对 PTS 的观察显示出许多几十纳米的颗粒散布于材料中 (图 3). 但电子 显微镜中观察到的颗粒通常较 Scherrer 方法的计算结果稍大, 且在几十纳米的范围内具有一 定的尺寸分布. 这种现象具有普遍性, 人们已对其中的原因作了分析 ${ }^{[3]}$.

表 1 的数据充分说明了当晶粒尺寸在几十纳米的范围内, PT 微晶表现出显著的小尺寸效 应. 随着晶粒尺寸的减小, PT 微晶的 $a$ 轴长度稍增大, $c$ 轴长度明显下降, $c / a$ 比显著降低, 铁 电四方相减弱. 结合图 4 可知, 随着 PT 晶粒减小, Curie 相变峰明显向低温方向移动, 相变潜 热大大降低, 相变温区逐渐展宽. $\mathrm{PbTiO}_{3}$ 的 Curie 相变本属一级相变, 在此表现出扩散相变 的性质. 这是由于对纳米微晶而言, 因相变温度随晶粒尺寸的减小而降低, 即使对同一尺寸 的 PT 微晶表现为一级相变, 而对具有一定尺寸分布的微晶集合宏观上也会表现出扩散相变 
表 $1 \mathrm{PbTiO}_{3}$ 微晶的晶粒尺寸、晶格参数和 Curie 相变峰的测试结果

\begin{tabular}{|c|c|c|c|c|c|}
\hline \multirow{2}{*}{ 材 } & \multicolumn{2}{|c|}{ 纯 $\mathrm{PbTiO}_{3}$} & \multicolumn{3}{|c|}{$\mathrm{PbTiO}_{3}+\mathrm{SiO}_{2}$ 复合物 } \\
\hline & 体材 & 微粉 & $85 \%$ PT & $73 \%$ PT & $42 \%$ PT \\
\hline 热处理条件 & $1250^{\circ} \mathrm{C}, 0.5 \mathrm{~h}$ & $600^{\circ} \mathrm{C}, 2 \mathrm{~h}$ & $800^{\circ} \mathrm{C}, 2 \mathrm{~h}$ & $800^{\circ} \mathrm{C}, 2 \mathrm{~h}$ & $800^{\circ} \mathrm{C}, 2 \mathrm{~h}$ \\
\hline PT 晶粒尺寸 (200)/nm & > 几百 & 66.4 & 31.8 & 27.3 & 22.5 \\
\hline$a$ 轴长度/nm & 0.3899 & 0.3903 & 0.3904 & 0.3918 & 0.3924 \\
\hline$c$ 轴长度/nm & 0.4154 & 0.4142 & 0.4126 & 0.4124 & 0.4096 \\
\hline$c / a$ 比 & 1.065 & 1.061 & 1.057 & 1.053 & 1.043 \\
\hline DSC 上居里峰/C & 490 & 488 & 483 & 481 & 475 \\
\hline
\end{tabular}

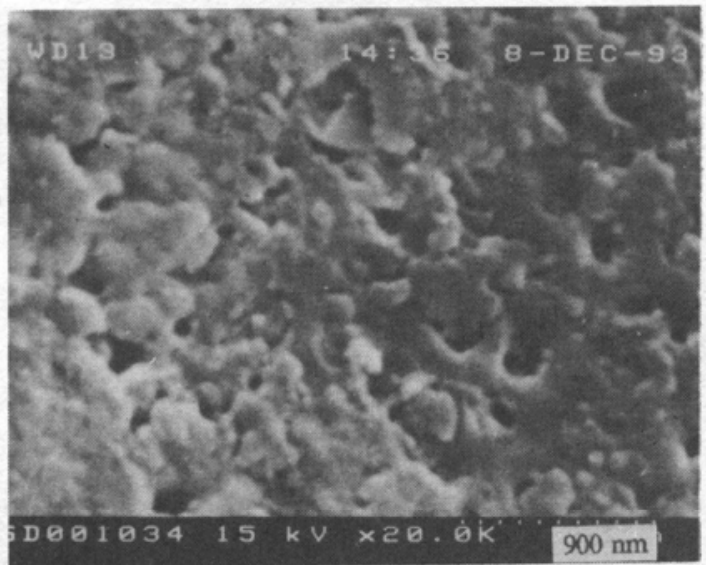

图 3 PTS 的 SEM 照片 (PT $\left.42 \%, 800{ }^{\circ} \mathrm{C}, 2 \mathrm{~h}\right)$

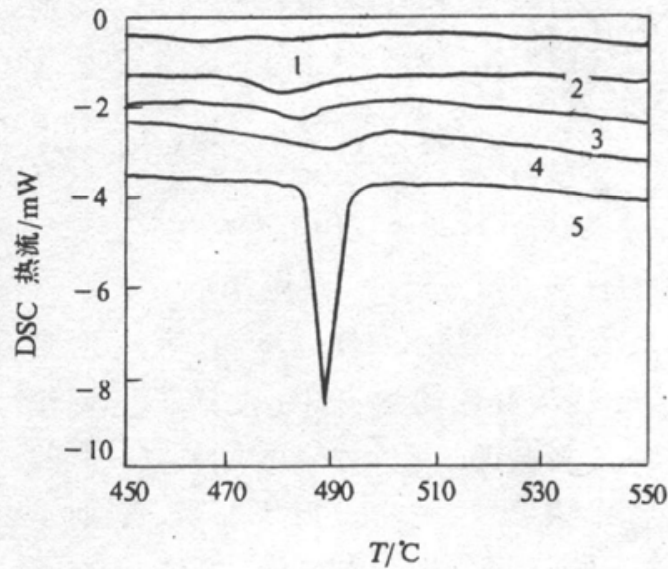

图 4 不同条件下获得的 PT 微晶的 DSC 曲线 1 为 $42 \% \mathrm{PT}, 800 \mathrm{C}, 2 \mathrm{~h} ; 2$ 为 $73 \% \mathrm{PT}, 800 \mathrm{C}, 2 \mathrm{~h}$; 3 为 $85 \% \mathrm{PT}, 800{ }^{\circ} \mathrm{C}, 2 \mathrm{~h} ; 4$ 为 PT 微粉, $600^{\circ} \mathrm{C}, 2 \mathrm{~h}$; 5 为 $\mathrm{PT}$ 微粉, $800^{\circ} \mathrm{C}, 2 \mathrm{~h}\left(10^{\circ} \mathrm{C} / \mathrm{min}\right)$

的性质. 近年来的理论研究表明, 在不考虑尺寸分布的情形下, 为降低体系自由能, $\mathrm{PbTiO}_{3}$ 微 晶体系也有可能存在着铁电相和顺电相共存的涨落现象. 另外, 从本文 DSC 曲 线上观察到 的随晶粒尺寸减小 Curie 峰的下降幅度并没有通过 Raman 光谱研究观察到的大 ${ }^{[3]}$.

\section{参 考 文 献}

1 Herzog A. Microcrystalline $\mathrm{BaTiO}_{3}$ by crystalliyation from glass. J Am Ceram Soc, 1964, 47(3): $107 \sim 115$

2 Borrelli N F, Layton M M. Electron-optic properties of transparent ferroelectric glass-œramic systems. IEEE Trans Electro Devic, 1969, 16(6): 511 514

3 Ishikawa K, Yoshikawa K, Okada N. Size effect on the ferroelectric phase transition in $\mathrm{PbTiO}_{3}$ ultrafine particles. Phys Rev, 1988, B37(10): $5852 \sim 5855$

4 姚 喜,张良荣. 精细复合功能材料. 物理, 1992, 21(2): 99 105

5 周歧发,李永祥,张良莹等. 陶瓷氧化物超微粉的制备. 科学通报, 1991，36(22): 1 751 1753

6 周歧发, 韩 玉,邹 秦等. PT-ER 精细复合材料的光学特性研究. 科学通报, 1992, 37(7): 665 667 